\title{
Pars Plana Vitrectomy With Silicone Oil Versus Sulphur Hexafluride Gas Tamponade For Idiopathic Macular Hole
}

Mohamed Saad Ibrahim ${ }^{1, *}$ MSc; Ahmed Hassan Barrada ${ }^{1}$ MD ; Mahmoud Mohamed $\mathrm{Ali}^{1} \mathrm{MD}$

\author{
*Corresponding Author: \\ Mohamed Saad Ibrahim \\ dr.mohamed_alabshihy@yahoo.com
}

Received for publication April 28, 2021; Accepted May 28, 2021; Published online May 28, 2021.

\begin{abstract}
Copyright The Authors published by Al-Azhar University, Faculty of Medicine, Cairo, Egypt. Users have the right to read, download, copy, distribute, print, search, or link to the full texts of articles under the following conditions: Creative Commons AttributionShare Alike 4.0 International Public License (CC BY-SA 4.0).

doi: 10.21608/aimj.2021.74262.1465

${ }^{1}$ Ophthalmology Department, Faculty of Medicine, Al-Azhar University, Cairo , Egypt.
\end{abstract}

\begin{abstract}
Background: A macular hole is a common macular disorder that impairs central vision significantly.

Aim of The Work: To compare between silicone oil (SOi) versus sulphur hexafluoride (SF6) as tamponading agent after pars plana vitrectomy (PPV) and internal limiting membrane (ILM) peeling in healing of idiopathic macular hole and improvement of visual acuity.

Patient and Methods: Patients with idiopathic full thickness macular hole (FTMH) were categorized into 2 groups, group 1(15 eyes) were managed with PPV and ILM peeling and (SF6) as atamponade, group 2 (15 eyes) were managed with PPV and ILM peeling and (SOi) as a tamponade. All patients' best-corrected visual acuity (BCVA), and optical coherence tomography (OCT) were followed up for six months.

Results: Six months postoperatively BCVA in LogMAR in the first (Gas) group improved from a baseline mean of 1.23 to 0.41 , gaining an improvement of $66.33 \%$, which is more than the improvement of the second (SOi) group from a baseline of 1.19 to 0.56 (53.72\%) so this difference was significant (P- value 0.026 (. Just two cases, (only one case in each group) (6.4\%) failed to close after six months. The success rate was $94 \%$, with no statistically significant difference.

Conclusion: Idiopathic (FTMH) was treated with (PPV) with ILM peeling . Both SF6 and (SOi) tamponades are similarly effective in anatomical and visual results, using (SOi) in macular hole surgery necessitates two surgeries: one for the initial vitrectomy and (SOi) injection, and another several weeks later to extract the oil.
\end{abstract}

Keywords: tamponade; pars plana vitrectomy; macular hole.

Disclosure: The authors have no financial interest to declare in relation to the content of this article. The Article Processing Charge was paid for by the authors. Authorship: All authors have a substantial contribution to the article.

INTRODUCTION

A macular hole is a common macular disorder that impairs central vision significantly ${ }^{1}$

A macular hole is a neurosensory retinal anatomical discontinuity that occurs in the center of the macula and fovea . $^{2}$

The prevalence of full-thickness macular hole $(\mathrm{MH})$ is 7.9 eyes per 100,000 people per year, and the disease has a major effect on the quality of life of those who are affected. ${ }^{3}$

A macular hole was divided according to cause into primary (idiopathic) or secondary (trauma, high myopia, after successful retinal detachment surgery) and by presence or absence of vitreous attachment. They also classified into small $(<250 \mu \mathrm{m})$, medium $(>250 \mu \mathrm{m}<400 \mu \mathrm{m})$, and large $(>400 \mu \mathrm{m})$ based on horizontal macular hole diameter (MHD) width at the narrowest point of the hole. ${ }^{4}$

Hole closure rates are high, at over $90 \%$, but different factors, including the size of the hole, are known to reduce the success rate. Several surgical variations and adjunctive procedures have been described to improve hole closure rates including post-operative face down positioning, longer acting tamponade, surgical creation of ILM flaps, and other biological membranes and platelets. ${ }^{5}$

The standard treatment for FTMH is pars plana vitrectomy with internal limiting membrane peeling and intraocular gas tamponade, followed by several days in a face-down position ${ }^{6}$ Since then, several modifications of this technique have been developed to improve postoperative anatomical results and to achieve better visual acuity. This techniques include, classic inverted ILM flap technique ${ }^{7}$, or modified temporal inverted ILM flap technique ${ }^{8}$,especially in large MHs or in patients with high myopia, use of autologous platelet concentrate, anterior lens capsule use, and the use of different types of intraocular tamponading agents such as air, gas, and silicone oil (SOi) ${ }^{9}$

In a significant fraction of patients, visual acuity improvement after MH surgery persisted for the first three years after PPV and was sustained afterwards, and final BCVA associated with better preoperative BCVA and better postoperative OCT parameters.$^{10}$

In this study, we evaluated the efficacy and safety of SF6 gas and silicone oil as a tamponade after PPV and ILM peeling in treating patients with idiopathic FTMH. 


\section{PATIENTS AND METHODS}

A prospective interventional comparative clinical trial was conducted at Al-Azhar University Hospitals (Cairo, Egypt) from October 2018 to May 2020 and was followed up for 6 months. The study included two groups; each included 15 eyes, all of which had idiopathic full thickness macular hole with hole diameter more than 400um on (OCT).

Exclusion criteria: were significant media opacity preventing retinal view, presence of traction on the macula evidenced by OCT, previous ocular surgery, history of previous laser photocoagulation, IV injection or history of uveitis attacks, any chorioretinal disease or diabetic retinopathy, glaucoma or ocular hypertension, Patients with high myopia, cases of age related macular degenerations (AMD), Other causes of macular hole as myopic or traumatic macular hole.

A full ophthalmic history and assessment was performed to all patients, which included LogMAR (BCVA), anterior segment slit lamp, applanation fundus slit lamp biomicroscopy, and, tonometry. The diagnosis of a macular hole was made both clinically and using (OCT).

Surgical procedures: Thiry eyes were assigned to one of the following 2 groups according to the regimen used in the management idiopathic FTMH , each group consists of 15 eyes; Group 1 (GAS group); Patients were treated with vitrectomy, ILM peeling, and SF6 as a tamponade, as well as a oneweek post-operative face down position. Patients in Group 2 (SILICONE OIL) were treated with vitrectomy, ILM peeling, and silicone oil as a tamponade with no special post-operative position. Both groups do phacoemulsification in phakic eyes with vitrectomy to exclude lens status and results easily analysed. After 2-3 months, Group 2 had a second surgery to remove silicone oil.

All surgeries were performed under local anesthesia with sedation. Standard sterilization using PovidoneIodine $5 \%$ (Betadine ${ }^{\circledR}$ ) by lid swabbing and instillation in the conjunctival sac was done and the patient was draped.

23 G PPV with three-port sclerotomies were performed, followed by fluid air exchange, and ILM staining with brilliant blue dye. The target was to peel at least 2 disc-diameter ILM areas 360 degrees around the hole. Following that, air gas exchange was performed for the gas-treated eyes, a $20 \mathrm{~mL}$ syringe containing $20 \%$ SF6 gas was connected to an infusion cannula. Flute needle used to allow gas and air to escape from the superior temporal sclerotomy site while the assistant administered $17 \mathrm{~mL}$ via the infusion cannula. The vitreous cavity is filled with $20 \%$ SF6 during this process, after removal of sclerotomy ports their sites were tested for leakage before being sutured shut with Vicryl 7-0 sutures. Before being sutured shut with Vicryl 7-0 sutures, it was screened for leakage. If hypotony was detected at the end of the procedure, the remaining $3 \mathrm{~mL}$ of gas was injected through the pars plana with a $30 \mathrm{~g}$ needle to apply to the tamponade. After fluid air exchange, one of the upper two sclerotomies was used to inject silicone 1000, while the other sclerotomy was used to enable air to escape via a flute needle. At the conclusion of the procedure, subconjunctival cefuroxime and dexamethasone were injected. Following surgery, cyclopentolate $1 \%$ drops were used four times a day for two weeks and combined antibiotic-steroid drops (tobramycin $0.3 \%$ with dexamethasone $0.1 \%$ ) were used six times a day for four three weeks. Patients with gas-filled eyes were orderd to keep their prone position with faces down until 50 percent of the gas had been absorbed, or for at least one week. Patients with silicon-filled eyes were not told to take any special positions.

Follow-up: The day after surgery, one week later, one month later, three months later, and six months later, patients were evaluated. BCVA, IOP, slit lamp, and fundus exams were performed at the two-week, one-month, three-month, and six-month visits.

(OCT) was done at one month, then three months and six months postoperatively to confirm successful anatomic closure. During each postoperative visit, the foveal contour and the presence of photoreceptor line defects, and dissociated optic nerve fiber layer defect (DONFL) were estimated.

Postoperative foveal contour was characterized according to its cross-sectional morphology on spectral domain optical coherence tomography into the various categories, U-shape, $\mathrm{V}$-shape, W-shape (irregular), flat shaped.

Statistical analyses were performed by the IBM SPSS software package version 20.0 which analyse the data that was fed into the device. . All values are expressed as $\mathrm{MD} \pm \mathrm{SD}$. P-values less than 0.05 were accepted as significant.

Ethical approval: The study was accepted by the Ethics Board of Al-Azhar University. Informed written consent was obtained from every patient before being included in the study.

\section{RESULTS}

Group 1 (gas group) included 15 eyes from 15 patients (7 females and 8 males), while group 2 included 15 eyes from 15 patients ( 9 females and 6 males) (silicone oil group).The ages in group 1 ranged from 44 to 69 years $55.80 \pm 7.68$ (mean + SD), while the ages in group 2 ranged from 47 to 73 years $57.67 \pm 7.52$ (mean $+\mathrm{SD}$ ). In terms of age and gender, there was no statistically significant difference between the two study groups.

\section{Anatomical Success Rate:}

In the SF6 group, the mean preoperative BCVA ranged from $(\mathrm{CF}$ at $50 \mathrm{~cm})$ or 1.4 to $(6 / 60)$ or 1.00 , with a mean BCVA of 1.23 and an SD of 0.11. In the silicone oil group, the mean preoperative LogMAR BCVA ranged from (CF at $50 \mathrm{~cm}$ ) or 1.4 to $(6 / 60)$ 1.00, with a mean BCVA of 1.19 and an SD of 0.13. The size of hole base diameter in group 1(gas group) ranged from $433.0 \mu \mathrm{m}$ to $1682.0 \mu \mathrm{m} 850.0 \pm 403.8$ (mean $+\mathrm{SD}$ ), while the size of hole base diameter in group 2 (silicone group) ranged from $456.0 \mu \mathrm{m}$ to $1359.0 \mu \mathrm{m} 801.3 \pm 302.3$ (mean $+\mathrm{SD})$. 
At 6 months, twenty-eight (93.3\%) of the cases had closed anatomically, while only two ( just one case in each group) (6.4\%) had not anatomically closed. The success rate was 94 percent in gas-filled (14/15) eyes and 94 percent in silicone-filled (14/15) eyes, with no statistically significant difference. (Table 1).

\begin{tabular}{|c|c|c|c|c|c|c|}
\hline \multirow[t]{2}{*}{ OCT } & \multicolumn{2}{|c|}{$\begin{array}{c}\text { Gas group } \\
(\mathrm{n}=15)\end{array}$} & \multicolumn{2}{|c|}{$\begin{array}{l}\text { Silicone group } \\
(n=15)\end{array}$} & \multirow[t]{2}{*}{$x^{2}$} & \multirow[t]{2}{*}{${ }^{{ }^{M} C_{p}}$} \\
\hline & No. & $\%$ & No. & $\%$ & & \\
\hline $\begin{array}{l}\text { Post-operative lm } \\
\text { Flat healing } \\
\text { V shaped healing } \\
\text { U shaped healing } \\
\text { W shaped healing } \\
\text { Failure of healing }\end{array}$ & $\begin{array}{l}4 \\
7 \\
2 \\
1 \\
1\end{array}$ & $\begin{array}{l}26.7 \\
46.7 \\
13.3 \\
6.7 \\
6.7\end{array}$ & $\begin{array}{l}3 \\
8 \\
3 \\
0 \\
1 \\
\end{array}$ & $\begin{array}{l}20.0 \\
53.3 \\
20.0 \\
0.0 \\
6.7\end{array}$ & 1.717 & 1.000 \\
\hline $\begin{array}{l}\text { Post-operative 3ms } \\
\text { Flat healing } \\
\text { V shaped healing } \\
\text { U shaped healing } \\
\text { W shaped healing } \\
\text { Failure of healing }\end{array}$ & $\begin{array}{l}4 \\
5 \\
5 \\
0 \\
1\end{array}$ & $\begin{array}{l}26.7 \\
33.3 \\
33.3 \\
0.0 \\
6.7\end{array}$ & $\begin{array}{l}2 \\
4 \\
6 \\
2 \\
1\end{array}$ & $\begin{array}{l}13.3 \\
26.7 \\
40.0 \\
13.3 \\
6.7\end{array}$ & 4.723 & 0.540 \\
\hline $\begin{array}{l}\text { Post-operative } 6 \mathrm{~ms} \\
\text { Flat healing } \\
\text { V shaped healing } \\
\text { U shaped healing } \\
\text { W shaped healing } \\
\text { Failure of healing }\end{array}$ & $\begin{array}{l}4 \\
5 \\
5 \\
0 \\
1\end{array}$ & $\begin{array}{l}26.7 \\
33.3 \\
33.3 \\
0.0 \\
6.7\end{array}$ & $\begin{array}{l}2 \\
4 \\
6 \\
2 \\
1\end{array}$ & $\begin{array}{l}13.3 \\
26.7 \\
40.0 \\
13.3 \\
6.7\end{array}$ & 4.723 & 0.540 \\
\hline
\end{tabular}

MC: Monte Carlo

$\mathrm{P}$ : $\mathrm{p}$ value for comparing between the studied groups Table 1: Comparison between the two studied groups according to OCT.

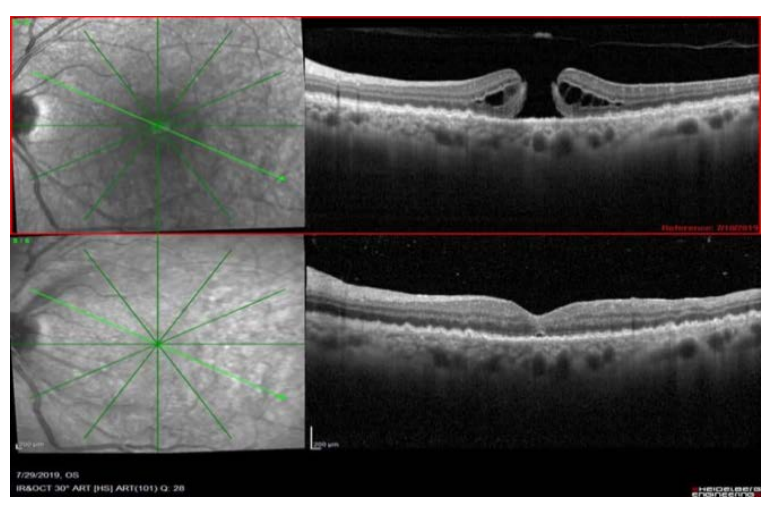

Fig. 1: OCT pre and post vitrectomy with ILM peeling show closure of hole and $\mathrm{U}$ - shaped healing in the SF6 group .

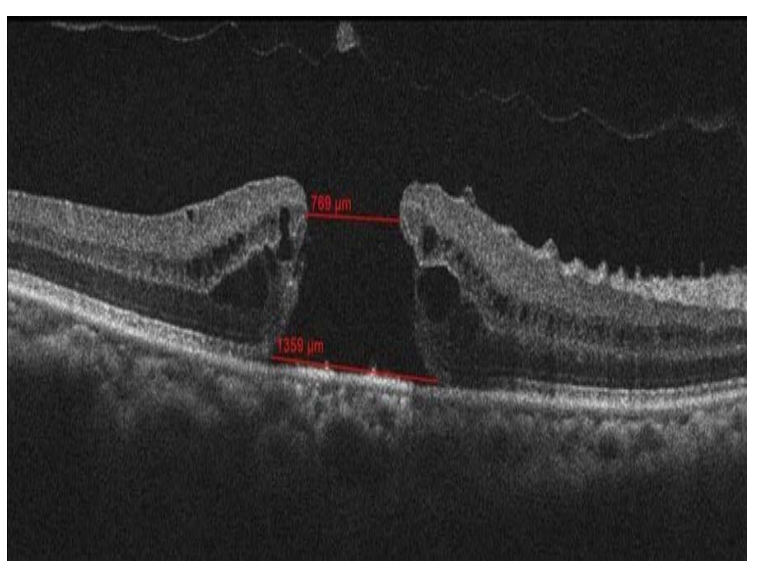

Fig. 2: OCT pre and post operative show closure of hole and $\mathrm{U}$ - shaped healing in the silicon oil group.

\section{Visual Outcomes :}

Comparing the BCVA of both groups; mean BCVA at 1month in the first group improved from a baseline mean of 1.23 to 0.71 , gaining an improvement of $42.29 \%$, which is more than the improvement of the second group from a baseline of 1.19 to 0.84 (29.63\%) but this difference was insignificant (P value 0.116). At 3 months in the first group improved from a baseline mean of 1.23 to 0.49 , gaining an improvement of $60.52 \%$, which is more than the improvement of the second group from a baseline of 1.19 to $0.60 \quad(49.58 \%)$ but this difference was insignificant (P value 0.056).At 6 months in the first group improved from a baseline mean of 1.23 to 0.41 , gaining an improvement of $66.33 \%$, which is more than the improvement of the second group from a baseline of 1.19 to $0.56 \quad(53.72 \%)$ so this difference was significant ( $\mathrm{P}$-value 0.026)(Table 2).

\begin{tabular}{|c|c|c|c|c|}
\hline $\begin{array}{c}\text { Visual Acuity } \\
\text { (log mar) }\end{array}$ & $\begin{array}{c}\begin{array}{c}\text { Gas } \\
(\mathrm{n}=15)\end{array} \\
\text { (15) }\end{array}$ & $\begin{array}{l}\begin{array}{c}\text { Silicon } \\
(\mathrm{n}=15)\end{array}\end{array}$ & $\mathrm{U}$ & p \\
\hline \multicolumn{5}{|l|}{ Preoperative } \\
\hline Min. - Max. & $1.0-1.40$ & $1.0-1.40$ & \multirow{3}{*}{95.000} & \multirow{3}{*}{0.486} \\
\hline Mean \pm SD. & $1.23 \pm 0.11$ & $1.19 \pm 0.13$ & & \\
\hline Median (IQR) & $1.20(1.20-1.30)$ & $1.20(1.10-1.30)$ & & \\
\hline \multirow{2}{*}{\multicolumn{5}{|c|}{$\begin{array}{l}\text { Postoperative } \\
1 \text { month }\end{array}$}} \\
\hline & & & & \\
\hline Min. - Max. & $0.35-1.05$ & $0.55-1.15$ & \multirow{3}{*}{74.000} & \multirow{3}{*}{0.116} \\
\hline Mean \pm SD. & $0.71 \pm 0.22$ & $0.84 \pm 0.22$ & & \\
\hline Median (IQR) & $0.75(0.55-0.85)$ & $0.75(0.70-1.05)$ & & \\
\hline \multicolumn{5}{|l|}{3 months } \\
\hline Min. - Max. & $0.30-0.85$ & $0.30-1.0$ & \multirow{3}{*}{66.000} & \multirow{3}{*}{0.056} \\
\hline Mean \pm SD. & $0.49 \pm 0.18$ & $0.60 \pm 0.17$ & & \\
\hline Median (IQR) & $0.45(0.35-0.53)$ & $0.60(0.50-0.70)$ & & \\
\hline \multicolumn{5}{|l|}{6 months } \\
\hline Min. - Max. & $0.20-0.85$ & $0.35-1.05$ & \multirow{3}{*}{$59.000^{\circ}$} & \multirow{3}{*}{0.026} \\
\hline Mean \pm SD. & $0.41 \pm 0.21$ & $0.56 \pm 0.19$ & & \\
\hline Median (IQR) & $0.30(0.30-0.50)$ & $0.55(0.40-0.65)$ & & \\
\hline
\end{tabular}

SD : standard deviation

Table 2: Comparison according to visual acuity log mar between the two studied groups.

In the SF6 group, the mean preoperative BCVA ranged from (CF at $50 \mathrm{~cm})$ or 1.4 to $(6 / 60)$ or 1.00 , with a mean BCVA of 1.23 and an SD of 0.11 . In the silicone oil group, the mean preoperative LogMAR BCVA ranged from CF at $50 \mathrm{~cm}$ (1.4) to 6/60 (1.00), with a mean BCVA of 1.19 and an SD of 0.13. The diameter of the hole base diameter in group 1 (gas group) ranged from $433.0 \mathrm{~m}$ to $1682.0 \mathrm{~m}$ (mean + $\mathrm{SD}$ ), whereas the diameter of the hole base diameter in group 2 (silicone group) ranged from $456.0 \mathrm{~m}$ to 1359.0m (mean + SD) (Figure 3).

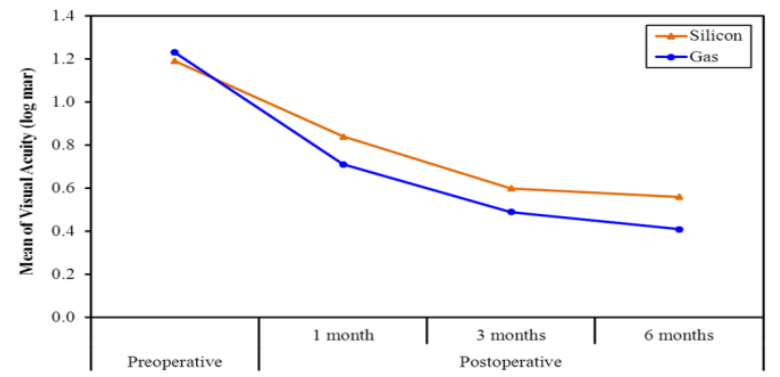

Fig. 3: Comparison between the two studied groups according to visual acuity log mar. 


\section{Comparison of IOP:}

Comparing the IOP of both groups; mean IOP at 1st month in the first group rise from a baseline mean of 15.2 to 19.6, gaining a rise of $32.98 \%$, While in the second group (a baseline of 15.27 to 21.13) gaining a rise of $14.36 \%$, and this difference was insignificant (P value 0.199). At 3rd months the first group was 17.0, with the second group returned to 17.07 to become close to baseline and this difference was insignificant ( $\mathrm{P}$ value 0.946 ). At the 6th month the first group was 16.00, with the second group 15.00 and this difference was also insignificant ( $P$ value 0.103) (Figure 4).

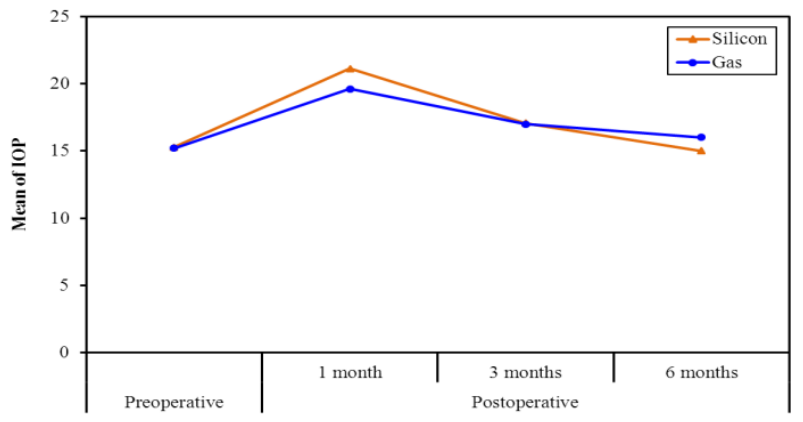

Fig. 4: Comparison between the two studied groups according to IOP.

\section{Adverse Events}

The macular hole failed to close in two cases (6.6 percent) (one case in each group). One patient in the gas-treated group had a high (IOP) on day one after surgery, which was managed medically for two weeks. There were no cases of endophthalmitis, choroidal haemorrhage, or retinal detachment in the report.

\section{DISCUSSION}

A prospective research was conducted on 30 patients' eyes, divided into two groups of 15 patients each. The first group ( the gas group ) received vitrectomy with ILM peeling and SF6 20\% tamponade, while the second group ( the silicone oil group ) received vitrectomy with ILM peeling and silicone oil as tamponade. Patients were recruited and enrolled at Al-Azhar University Hospitals in Cairo, Egypt, from October 2018 to May 2020, and were followed for six months.

In this study, we evaluated the efficacy and safety of SF6 gas and silicone oil as a tamponade after vitrectomy and ILM peeling in treating patients with idiopathic FTMH. The aim of the combined therapy was not only to achieve closure of hole, but also to observe the functional success and improvement of visual acuity and provide patient comfort postoperatively.

After one month of treatment, the closure rate for gas-treated eyes was 93.3 percent (14 of 15), and the closure rate for silicone oil tamponade was 93.3 percent (14 of 15). Idiopathic macular hole closure had a 93.3 percent primary success rate (28 of 30). The postoperative LogMAR BCVA in the SF6 group ranged from 1.4 to 1.00 at six months, with a mean BCVA of 1.23 and an SD of 0.11 , according to the visual results. Although the silicone oil group postoperative BCVA ranged from 1.4 to 1.00 at six months, the mean BCVA was 1.19 with an SD of 0.13 .

A t-test was used to assess postoperative BCVA in the two groups at six months. In the gas (SF6) group, there was a statistically significant difference in BCVA. This may be due to many factors, such as SOi being used for larger macular holes, resulting in more retinal pigment epithelium (RPE) and photoreceptor damage, and the SOi having a lower surface tension than the gas.

As compared to previous studies of traumatic macular hole, as well as myopic or idiopathic hole, the anatomical closure rates in this study were favourable. This could be because traumatic macular holes close faster than myopic or idiopathic holes due to the patient's younger age, earlier diagnosis, and the fact that traumatic macular holes have a higher average normal closure rate than myopic or idiopathic holes. ${ }^{11}$

As regard our study the IOP of gas group before operation ranged from $10 \mathrm{mmHg}$ to $19 \mathrm{mmHg}$ with mean IOP was 15.20 with a SD \pm 2.76 . While in silicone group before operation ranged from $11 \mathrm{mmHg}$ to $19 \mathrm{mmHg}$ with mean IOP was 15.27 with a $\mathrm{SD} \pm 2.31$. At one month after surgery, the mean IOP in the gas group was 19.60, with an insignificant improvement of 32.98 percent, but the mean IOP in the silicone group was 21.13, with a substantial increase in IOP of 40.41 percent. At three months after surgery, the mean IOP in the gas group was 17.0, with a non-significant change of 14.12 percent, and the silicone group had a non-significant difference of 17.07, with a non-significant change of 14.36 percent. At six months post operative there is insignificant difference between both groups.

Increased IOP was measured in $2.6 \%$ of the cases, which is much lower compared to $20-21 \%$ reported by others on the first post-operative day ${ }^{12}$, the IOP could be regulated with anti-glaucoma agents. SF6 belongs to a group of expansile gases used as tamponade agents, and its concentration can vary in different studies.$^{13}$

Heath and Rahman used a 20 percent SF6 concentration in all cases, which is considered nonexpansile. As a result, the incidence of increased IOP post-operatively may be much lower or similar to studies showing no post-operative IOP spike. ${ }^{14}$ The risk for post-operative hypotony reported to occur with $23 \mathrm{G}$ vitrectomy ${ }^{15}$, which was not apparent in our study.

Rebecca et al , reported that In eyes that underwent silicone oil implantation, $46.82 \%$ had a rise in IOP by 1 month, $18.25 \%$ between 1 and 3 months and $25.39 \%$ from 3 months after transconjunctival sutureless vitrectomy. Similarly, 57.69 percent of eyes with air/gas implantation had an increase in IOP after one month, 30.76 percent between one and three months, and 3.8 percent after three months of (TSV). 50 percent of eyes with other PPV procedures without tamponade had an increase in IOP after one month, 18.18 percent between one and three months, 
and 18.18 percent after three months after TSV. As a result, regardless of the tamponading agent used, the peak IOP was always seen in the first month. ${ }^{16}$

The first improvement in the macula after ILM peeling is postoperative swelling of the arcuate retinal nerve fibre layer (SANFL), which disappears within 3 months; the swelling is not visible on biomicroscopic fundus inspection but appears as hypoautofluorescent arcuate striae in the macular area on infrared and autofluorescence imaging. SANFL is accompanied by (DONFL), which is barely visible on fundus inspection and corresponds to "dimples" in the inner retinal layers on OCT, despite the fact that these changes (DONFL) have no effect on postoperative visual acuity or even microperimetry changes. ${ }^{17}$

In our study, (DONFL) appearance was noticed in $75 \%$ of group I cases and in $60 \%$ of group II cases at 6 months postoperatively. There was no statistically significant effect of DONFL appearance on postoperative BCVA in both groups.

DONFL was more common in Group I (gas group) than in Group II (silicon oil group) (not statistically significant), and DONFL was only visible on the temporal side, suggesting that it was caused by ILM peeling. Functionally, the visual acuity in cases with and without a DONFL appearance was not significantly different. Pre- and post-operative Bscans and C-scans en face SD-OCT were used by Alkabes et al to assess the characteristic OCT-pattern of DONFL in 36 eyes of 36 patients who underwent vitrectomy for idiopathic $\mathrm{MH}$. At three months after surgery, a face SD-OCT (Cirrus HD-OCT) revealed DONFL presence in 100 percent of the eyes.. ${ }^{18}$

\section{CONCLUSION}

For nonclosing cases of idiopathic FTMH, PPV with ILM peeling should be performed. Both gas and (SOi) tamponades are effective in anatomical and visual outcomes, but in macular hole surgery, gas (SF6) tamponade provides much faster visual rehabilitation than (SOi), despite the fact that (SOi) necessitates two separate surgeries: one for the initial vitrectomy and injection of oil, and another surgery many weeks later to remove it which increase patients financial cost.

\section{REFERENCES}

1. Ruoan Han , Chenxi Zhang, Xinyu Zhao1, Youxin Chen1, Treatment of primary full-thickness macular hole by intravitreal injection of expansile gas, The Royal College of Ophthalmologists. 2018; 41433018-0098-z.

2. Barak Y, Ihnen MA, Schaal S. Spectral domain optical coherence tomography in the diagnosis and management of vitreoretinal interface pathologies. $J$ Ophthalmol. 2012; 1012: 876472.
3. Forsaa VA, Lindtjorn B, Kvaloy JT, Epidemiology and morphology of full-thickness macular holes. Acta Ophthalmol. 2018;96:397e404.

4. Steel DHW, Lotery AJ . Idiopathic vitreomacular traction and macular hole: a comprehensive review of pathophysiology, diagnosis, and treatment. Eye .2016; 27:S1-S21.

5. Madi HA, Masri I, Steel DH. Optimal management of idiopathic macular holes. Clin Ophthalmol. 2016;10:97-116.

6. M. Parravano, F. Giansanti, C. M. Eandi, Y. C. Yap, S. Rizzo, and G. Virgili, "Vitrectomy for idiopathic macular hole," Cochrane Database of Systematic Reviews2015; vol. 5, p. CD009080.

7. Michalewska Z, Michalewski J, Adelman RA, et al. Inverted internal limiting membrane flap technique for large macular holes. Ophthalmology. 2010; 117(10): 2018-25.

8. Takai Y, Tanito M, Sugihara . Temporal inverted internal limiting membrane flap technique for macular hole patients unable to maintain postoperative prone positioning.Retin Cases Brief Rep .2016; 10(4): 323-6.

9. Yepez JB, Murati FA, De Yepez JF, Anterior lens capsule in the management of chronic full-thickness macular hole. Retin Cases Brief Rep. Epub ahead of print 28 December 2016.

10. Ophthalmology Retina by the American Academy of Ophthalmology,2019 : volume : 60 Issue : 3. pages 369-376.

11. Oh H. Idiopathic macular hole. Developments in Ophthalmology. 2014;54:150-8.

12. Wong, R.; Gupta, B.; Williamson, T.H.; Laidlaw, D.A. Day 1 postoperative intraocular pressure spike in vitreoretinal surgery (VDOP1). Acta Ophthalmol. 2011; 89, 365-8.

13. Kannan, N.B.; Adenuga, O.O.; Kumar, K.; Ramasamy, K. Outcome of 2 cc pure sulfur hexafluoride gas tamponade for macular hole surgery. BMC Ophthalmol. 2016; 16, 56.

14. Heath, G.; Rahman, R. Combined 23-gauge, sutureless transconjunctival vitrectomy with phacoemulsification without face down posturing for the repair of idiopathic macular holes. Eye. 2010; 24, 214-20.

15. Gosse, E.; Newsom, R.; Hall, P.; Lochhead, J. Changes in Day 1 Post-Operative Intraocular Pressure Following Sutureless 23-Gauge and 
Conventional 20-Gauge Pars Plana Vitrectomy. Open Ophthalmol. J. 2013; 7, 42-7.

16. Rebecca Varkey, UG Unnikrishnan, Natasha Radhakrishnann, Incidence and risk factors for intraocular pressure rise after transconjunctival vitrectomy, Year: 2020 | Volume : 68 | Issue : 5 | Page : 812-817.

17. Pichi F, Lembo A, Morara M, Veronese C, Alkabes $\mathrm{M}$, Nucci P, et al. Early and late inner retinal changes after inner limiting membrane peeling. Int Ophthalmol. 2014; 34(2):437-46.

18. Alkabes M, Salinas C, Vitale L, Burés-Jelstrup A, Nucci P, Mateo C. En face optical coherence tomography of inner retinal defects after internal limiting membrane peeling for idiopathic macular hole. Invest Ophthalmol Vis Sci. 2011; 52(11):834955. 